\title{
Characterization of Plasmid DNA Location within Chitosan/PLGA/pDNA Nanoparticle Complexes Designed for Gene Delivery
}

\author{
Hali Bordelon, ${ }^{1}$ Alexandru S. Biris, ${ }^{2}$ Cristina M. Sabliov, ${ }^{1}$ and W. Todd Monroe ${ }^{1}$ \\ ${ }^{1}$ Biological and Agricultural Engineering Department, Louisiana State University Agricultural Center, \\ Rm. 149 E.B. Doran Bldg., Baton Rouge, LA 70803, USA \\ ${ }^{2}$ Nanotechnology Center, Applied Science Department, University of Arkansas at Little Rock, Little Rock, AR 72211, USA
}

Correspondence should be addressed to W. Todd Monroe, tmonroe@lsu.edu

Received 2 June 2010; Accepted 25 July 2010

Academic Editor: Lu Sun

Copyright (C) 2011 Hali Bordelon et al. This is an open access article distributed under the Creative Commons Attribution License, which permits unrestricted use, distribution, and reproduction in any medium, provided the original work is properly cited.

\begin{abstract}
Poly(D,L-lactide-co-glycolide-) (PLGA-)chitosan nanoparticles are becoming an increasingly common choice for the delivery of nucleic acids to cells for various genetic manipulation techniques. These particles are biocompatible, with tunable size and surface properties, possessing an overall positive charge that promotes complex formation with negatively charged nucleic acids. This study examines properties of the PLGA-chitosan nanoparticle/plasmid DNA complex after formation. Specifically, the study aims to determine the optimal ratio of plasmid DNA:nanoparticles for nucleic acid delivery purposes and to elucidate the location of the pDNA within these complexes. Such characterization will be necessary for the adoption of these formulations in a clinical setting. The ability of PLGA-chitosan nanoparticles to form complexes with pDNA was evaluated by using the fluorescent intercalating due OliGreen to label free plasmid DNA. By monitoring the fluorescence at different plasmid: nanoparticle ratios, the ideal plasmid:nanoparticle ration for complete complexation of plasmid was determined to be 1:50. Surface-Enhanced Raman Spectroscopy and gel digest studies suggested that even at these optimal complexation ratios, a portion of the plasmid DNA was located on the outer complex surface. This knowledge will facilitate future investigations into the functionality of the system in vitro and in vivo.
\end{abstract}

\section{Introduction}

Successful gene delivery and expression remains a significant hurdle that must be overcome before genetic therapies gain clinical acceptance. As such, there is currently much emphasis on further developing nucleic acid delivery systems, which can be classified into three categories: physical injection, viral vectors, and nonviral vectors [1]. Direct injection of genetic material is locally effective in tissues such as skin and muscle, but it can be invasive for more interior tissues, and it requires significant involvement of medical personnel during clinical administration [2]. Viral vectors have been effective in gene delivery, but significant immunological effects in patients have severely hampered their use [1-3]. Nonviral vectors include liposomes, lipid reagents, and polymeric nanoparticles (NPs). Because of their biocompatibility and ability to reach more interior target tissues, these delivery systems have the potential to circumvent the issues surrounding both direct injection and viral vectors $[1,2,4-6]$. Polymeric NPs have an advantage over both liposomes and lipid reagents because of their ease of manipulation, control over DNA release profiles, and biological stability in vivo [7]. In addition, the properties of these NPs can be easily modified, for example, by addition of functional polymer groups to increase uptake or improve release of cargo genetic material $[4,5,8]$.

One of the major drawbacks to gene encapsulation in synthetic polymers for delivery using bottom-up techniques is the incorporation of plasmid DNA (pDNA) into the particles during the synthesis phase. The encapsulation method requires the pDNA to be in contact with organic solvents, decreasing the chance for successful cellular delivery[9]. Of the polymeric NPs, delivery systems based on chemically modified cationic polymers have proven to be most effective 
[7, 10-12]. Kumar et al. developed cationically modified PLGA NPs which were designed to bind to pDNA postsynthesis in an attempt to reduce its degradation prior to delivery. In their study, a PVA-chitosan blend was used to stabilize PLGA NPs and to provide a positively charged surface for pDNA association $[13,14]$. Many reports have emphasized the use of the cationic polysaccharide chitosan to readily complex with negatively charged pDNA [10,15-17]. Kasturi et al. found that pDNA encapsulated within PLGA leads to significant confinement of the complexes within endolysosomes, yet the application of chitosan to the surface of the NPs was projected to improve pDNA-NP release from the endolysosomes and offer a more protected path through the cytoplasm to the nucleus [18].

There are several reports on various aspects of chitosanPLGA NPs for pDNA delivery, focusing on preparation methods, the final characteristics of synthesized NPs, and their behavior in vivo (i.e., cytotoxicity and transfection efficiency) [15-17, 19-21]. The ability of NPs to limit nuclease degradation of encapsulated pDNA has also been well documented $[4,21-23]$. There is a gap in information regarding particle characteristics and their effect on DNA binding which has been addressed only recently [24-26]. Surface images have been captured by scanning probe microscopy, a newer technique applied to this topic, yet are only capable of verifying morphology of the occurring particle-DNA complexes and not the degree of DNA encapsulation[24]. Studies have demonstrated that modification of particle characteristics such as particle size and overall surface charge can directly affect the amount and avidity of nucleic acid binding [27]. Variations in chitosan type and content, PLGA type, and particle preparation methods (i.e., centrifugation, freeze-drying) have been used to change these NP characteristics and have been shown to directly affect the binding efficiencies of the particles to nucleic acids such as antisense DNA oligonucleotides $[24,25]$ and small interfering RNAs [26]. Good manufacturing practices in pharmaceutical development require a thorough characterization of polymeric nanoparticles both before and after drug loading. The biological and chemical characteristics of nanoparticles can be altered by nucleic acid binding and must be completely understood before these delivery systems can be useful clinically [28]. Despite recent advancements in the synthesis and characterization of polymeric nanoparticles as improved drug delivery systems, the location of DNA in the particle-DNA complexes remains unknown.

It is the intent of this study to synthesize cationic chitosan-PLGA NPs designed for pDNA delivery, to verify the physiochemical properties of the particles while exploring their effect on pDNA binding at varied ratios, and ultimately to identify the location of DNA relative to the particles in the pDNA-NP complex. Methods used to characterize the complex include dynamic light scattering (DLS), scanning electron microscopy (SEM), and gel electrophoresis complexation and digestion assays. A newer technique, Surfaced-Enhanced Raman Spectroscopy (SERS), was utilized to detect pDNA location within the complexes. The final aim of this study is to provide further insight into an NP delivery system designed for plasmid DNA delivery that is completely characterized with respect to DNA location in the polymer-DNA complex. This knowledge will be necessary for development of NP-based genetic therapy formulations.

\section{Materials}

PLGA (Poly(DL-lactide-co-glycolide)) (50:50 (LA:GA), MW (40,000-75,000)) was purchased from Sigma Aldrich. PVA (Polyvinyl Alcohol) (Sigma, MW 9000-10,000) was obtained from Polysciences, Inc. Protasan chitosan chloride (>75-90 deactylate, $150-4000 \mathrm{kDa}$ ) was purchased from NovaMatrix Inc. (Norway). Ethyl Acetate HPLC/ACS grade was acquired from Fisher Scientific (Pittsburg, PA). The nanopure water $\left(\mathrm{ddH}_{2} \mathrm{O}\right)$ used in dialysis was prepared using a Nanopure Diamond (Barnstead) water purification system. Nylon closures were purchased from Fisher Scientific (Pittsburg, PA). pAcGFP1-N1 plasmid was obtained from Clontech Corporation (Mountain View, CA). Quant-IT OliGreen ssDNA Reagent and SYBR Gold nucleic acid stain were purchased from Invitrogen Corporation (Grand Island, NY). Fluorescence measurements were performed using Plastibrand PMMA cuvettes $(1.5 \mathrm{ml})$ purchased from Sigma Aldrich (St. Louis, MO) and measured using the Perkin Elmer LS 55 Luminescence Spectrometer (Waltham, MA). ReadyAgarose Mini Gels (1\% Agarose, 8 wells) were purchased from Bio-Rad Laboratories (Hercules, CA). Chitosanase (Streptomyces) and lysozyme (chicken egg white) were purchased from EMD Biosciences (San Diego, CA), and DNase I was purchased from New England Biolabs (Ipswich, MA)

\section{Methods}

3.1. PLGA Nanoparticle Synthesis. An organic phase was made by dissolving $200 \mathrm{mg}$ PLGA in $10 \mathrm{ml}$ of ethyl acetate at room temperature, stirring for 2 hours at $400 \mathrm{rpm}$ using a C25KC shaking incubator (New Brunswick Scientific, Edison, NJ). An aqueous phase was made by dissolving $100 \mathrm{mg}$ PVA in $10 \mathrm{ml}$ of $0.1 \mathrm{mM}$ ethyl acetate water solution at $75^{\circ} \mathrm{C}$ for $5 \mathrm{~min}$, under stirring. Following cooling, $30 \mathrm{mg}$ chitosan chloride was added to the PVA solution, and the mixture was stirred for 15-30 min at $1000 \mathrm{rpm}$ on the vortex mixer. The solution was allowed to degas at room temperature until clear. Following this step, $2.5 \mathrm{ml}$ of organic phase was added dropwise to $10 \mathrm{ml}$ aqueous phase under stirring at 14,000 rpm with the Ultra-Turrax T25 homogenizer T18 basic (Ika Works Inc., Wilmington, NC). Then, the emulsion was homogenized for extra 10 minutes and sonicated while in an ice bath for $10 \mathrm{~min}$ at $40 \%$ amplitude, 2 seconds on and 2 seconds off using a sonicator model CV33 (Vibracell, Sonic \& Materials Inc., Denbury CT). The solvent was evaporated with a Buchi rotovap R-124 (Buchi Analytical Inc., New Castle DE) set at $30^{\circ} \mathrm{C}$, under 40 psi vacuum and a nitrogen flow at $100 \mathrm{kpa}$ for $10 \mathrm{~min}$. A $40 \mathrm{ul}$ sample was removed and used for DLS analysis. The rest of the sample underwent purification by dialysis, followed by freeze-drying. 
3.2. Purification. The sample was purified by dialysis, using 100,000 MWCO, $1.8 \mathrm{ml} / \mathrm{cm}$ dialysis tubing (Fischer Scientific, Pittsburgh, PA), against distilled water. Dialysis was run for 8 hours, changing water every 2 hours. The particle solution was removed from inside the tube and placed into borosilicate vials with trehalose ( $2: 1 \mathrm{w} / \mathrm{w}$ trehalose:particles) added prior to freeze-drying.

3.3. Freeze-Drying. While freeze-dryer temperature was allowed to drop to $\sim-35^{\circ} \mathrm{C}$, the sample was prepared by approximate particle weight volume as $4.7 \mathrm{mg} / \mathrm{ml}$. Enough trehalose was added to empty, clean borosilicate vials to achieve a $1: 1$ or $1: 2$ trehalose-to-particle ratio. The appropriate volume of purified particle solution was added to the vial and mixed gently until trehalose was dissolved. The vials containing $1: 2 \mathrm{w} / \mathrm{w}$ particles:trehalose mixtures were frozen at $-80^{\circ} \mathrm{C}$ in an angled rack for 2 hours. Next, the sample vials were connected to the Labconco freeze dryer (FreeZone 2.5 Plus) and allowed to freeze-dry at $-35^{\circ} \mathrm{C}$ for 48 hours. The obtained powder was stored under refrigerated conditions until further use.

3.4. Chi/PLGA- pDNA Complex Formation. $0.5 \mathrm{mg}$ of dried $\mathrm{NP}$ was weighed out and combined with $0.5 \mathrm{ml}$ of water. The solution was vortexed for 15 minutes at $1000 \mathrm{rpm}$. NPs and pDNA were combined in Millipore water, vortexed for 30 seconds, and allowed to complex at $\mathrm{pDNA} / \mathrm{NP}$ ratios of $1: 1$, $1: 10,1: 20,1: 50$, and $1: 100$ by weight at room temperature undisturbed for 1 hour.

3.5. DNA Entrapment. To each sample, $25 \mathrm{ul}$ of OliGreen (OG) fluorescent nucleic acid stain (1X concentration in water per manufacturer's recommendations) was added to complexes containing a total of $4 \mathrm{ng}$ of pDNA and incubated at room temperature in a dark space for 5 minutes in a $1.5 \mathrm{ml}$ cuvette. After incubation, the total volume in the cuvette was raised to $500 \mathrm{ul}$ using Millipore water. The relative fluorescence was determined using $490 \mathrm{~nm}$ excitation and $520 \mathrm{~nm}$ emission wavelengths in an LS55B Luminescence Spectrophotometer. Complexes were analyzed in pDNA/NP ratios of $1: 1,1: 10,1: 20,1: 50$, and $1: 100(\mathrm{w} / \mathrm{w})$.

3.6. Electrophoretic Mobility. Electrophoretic mobility of pDNA-NP complexes was determined by gel electrophoresis. $100 \mathrm{ng}$ of pDNA $(10 \mathrm{ng} / \mathrm{ul})$ in similar pDNA/NP ratios described previously was mixed with $4 \mathrm{ul}$ of loading buffer, loaded into agarose gels $(1 \% \mathrm{w} / \mathrm{v})$ and run in TAE buffer at $6 \mathrm{~V} / \mathrm{cm}$ for 60 minutes, followed by staining with SYBR Gold for 15 minutes and visualization on a transilluminator.

3.7. Particle Size, Size Distribution, and Zeta Potential. NPs and pDNA-NP complexes were characterized in terms of size, size distribution, and zeta potential by dynamic light scattering (DLS) using the Malvern Zetasizer Nano ZS (Malvern Instruments Inc., Southborough, MA). In all cases, a volume of $1.3 \mathrm{ml}$ of each sample at a concentration of $0.3 \mathrm{mg} / \mathrm{ml}$ was placed in a polystyrene cuvette and the measurements were performed at $25^{\circ} \mathrm{C}$. The viscosity and refraction index were set equal to those specific to water. Zeta potential was measured with a disposable capillary cell with a volume of $1 \mathrm{ml}$ after purification. The mean values of size and PDI were determined using a monomodal distribution.

3.8. Enzymatic Digestion Assays. DNase I was used to digest pDNA not internalized within the NP complex whereas chitosanase and lysozyme were used to digest the NP polymers. DNase I (1U) was added to free pDNA and pDNA-NP complexes $(1: 50 \mathrm{pDNA} / \mathrm{NP})$ in the supplied DNase buffer, followed by incubation for 10 minutes at $37^{\circ} \mathrm{C}$. Plasmid digestion was analyzed via electrophoresis as described above. A chitosanase/lysozyme solution was prepared by combining $80 \mathrm{ul}$ chitosanase $(0.25 \mathrm{U} / \mathrm{mL})$ with $20 \mathrm{ul}$ of lysozyme $(100 \mathrm{U} / \mathrm{mL}$ in $50 \mathrm{mM}$ sodium acetate buffer) similar to that used to degrade chitosan NPs reported by Mao et al. [22]. $5 \mathrm{ul}$ of the enzyme cocktail was added to pDNA and complexes $(1: 50 \mathrm{pDNA} / \mathrm{NP})$ and incubated at $37^{\circ} \mathrm{C}$ for 18 hours. Samples were analyzed for enzymatic digestion via electrophoresis as described above.

3.9. pDNA Localization Using Surface-Enhanced Raman Spectroscopy. For the SERS experiments, silver NPs of 10 to $20 \mathrm{~nm}$ diameter were prepared first by citrate or borohydride mediated reduction of chloroauric acid or silver nitrate, respectively, based on the following protocol. Sodium borohydrate was added gradually to water followed by sodium citrate and by $\mathrm{AgNO}_{2}$ (dropwise) under slow stirring. Polyvinyl pyrrolidone (PVP) was added to the solution, and the mixture was stirred for 30 minutes. The resulting product was a goldish yellowish solution in color. The high magnification TEM image of the silver nanoparticles (SNPs) is shown in Figure 1(a).

The pDNA-NP complex suspension was mixed in equal parts with the SNP colloidal suspension under stirring for 2 hours over ice. A few droplets of the final mixture were deposited on a glass slide for Raman analysis. The overall analysis schematic is shown in Figure 1(b). Raman scattering studies were done at room temperature with a $633 \mathrm{~nm}(1.96 \mathrm{eV})$ laser excitation with a Horiba Jobin Yvon LabRam HR800 spectrometer equipped with a chargecoupled detector and two grating systems: 600 and 1800 lines $/ \mathrm{mm}$. The laser beam intensity measured at the sample was kept at $10 \mathrm{~mW}$, and the acquisition time was 5 seconds per sample. An Olympus microscope focused the incident beam to a spot size of $<1 \mu \mathrm{m}^{2}$, and the backscattered light was collected $180^{\circ}$ from the direction of incidence. Raman shifts were calibrated on a silicon wafer at the $520 \mathrm{~cm}^{-1}$ peak.

\section{Results}

4.1. Particle Size, Size Distribution, and Zeta Potential. Particle size analysis by DLS was completed for a range of pDNA-NP complex ratios. As shown in Figure 2(a), the size of the complexes was compared as a function of pDNA/NP ratios ranging from 0 (no DNA) to $1(\mathrm{w} / \mathrm{w})$. The effect of complexing NPs with pDNA binding at varied ratios produced particle sizes that increased with increasing 


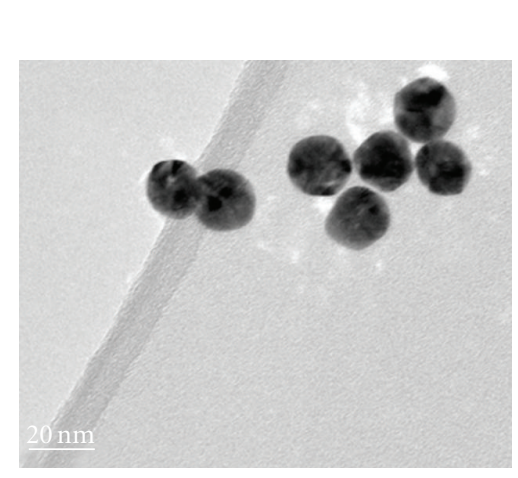

(a)

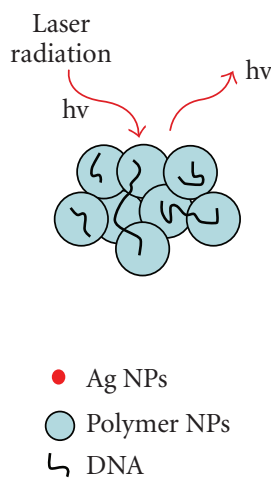

Polymer NPs
$\zeta$ DNA

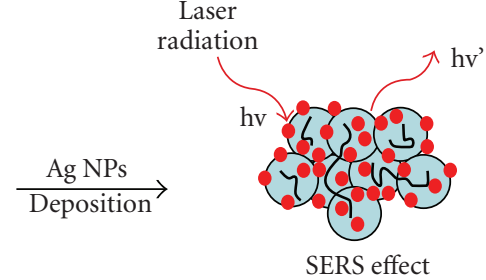

(b)

FIgURE 1: (a) High resolution TEM images of the Ag nanoparticles used for the SERS experiments. (b) The schematic of the Raman and SERS analysis of the DNA-Polymer NP mixtures.

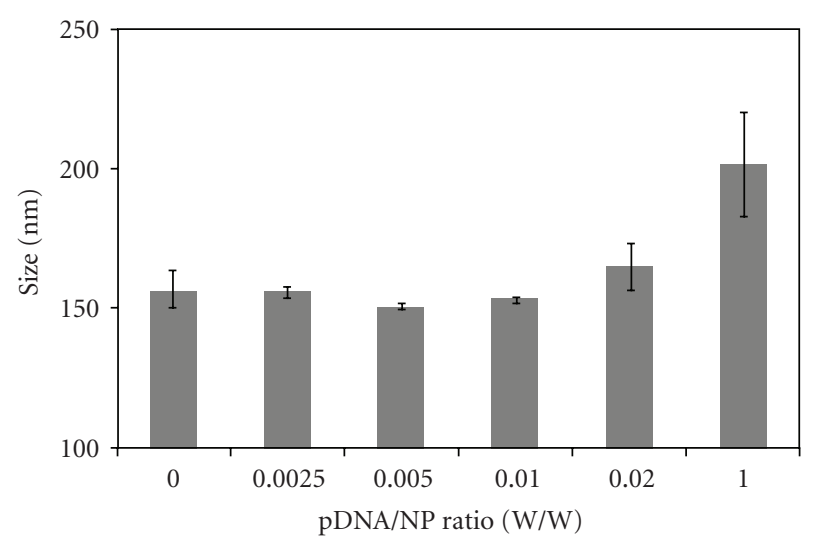

(a)

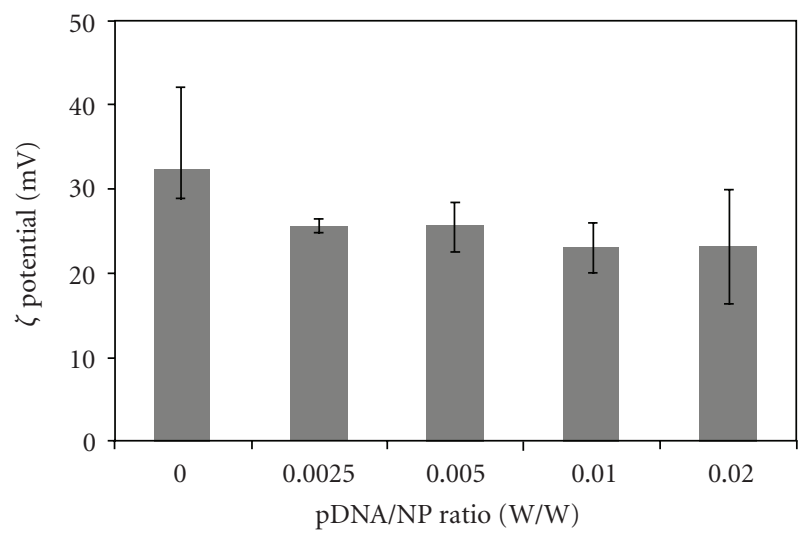

(b)

Figure 2: Size (a) and zeta potential (b) of pDNA-NP complexes, as a function of $\mathrm{pDNA} / \mathrm{NP}$ ratios $(n=3$; mean \pm standard error $)$.

pDNA/NP ratio. All pDNA-NP complexes remained less than $200 \mathrm{~nm}$ in size for $1: 400,1: 200,1: 100,1: 50,1: 10$, and $1: 1 \mathrm{w} / \mathrm{w}$ pDNA/NP.

Zeta potential analysis, shown in Figure 2(b), performed on the same pDNA-NP complexes indicates that the complexes were positively charged with a zeta potential higher

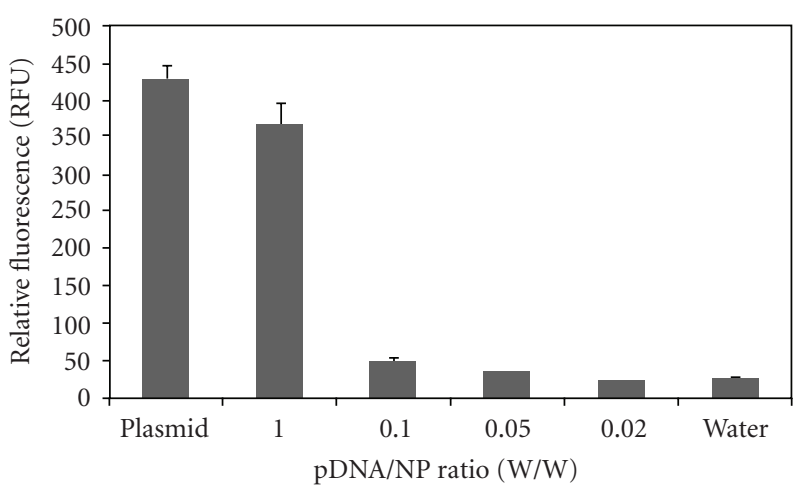

FIgure 3: The relative fluorescence of pDNA in each sample is dependent on the quantity of NPs in each complex. Maximum fluorescence is exhibited by free pDNA. Decreasing pDNA/NP ratios yield decreasing fluorescence intensities $(n=5$; mean \pm standard error).

than $20 \mathrm{mV}$ at all ratios studied. The pDNA-NP complexes at $1: 1$ ratio were not further analyzed because their larger diameter is not practical for cell delivery and a much larger amount of material would be required for accurate zeta potential analysis. As expected, adding more negatively charged pDNA decreased the zeta potential of the DNA-NP complexes.

\subsection{DNA Entrapment and Electrophoretic Mobility Studies.} The complex formation of pDNA and NPs was confirmed by fluorescence and gel electrophoresis. The relative fluorescence from the OliGreen nucleic acid stain is an indication of free, or incompletely complexed, DNA within the sample. Maximum fluorescence is exhibited by the sample that contained only free plasmid in water. As seen in Figure 3, a decrease in the pDNA/NP ratio corresponds to a decrease in fluorescence intensity. Such a decrease in fluorescence indicates that the pDNA is complexed to the point that DNA dye association and fluorescence are prevented. At a pDNA/NP ratio of 0.02 , a $94 \%$ decrease in fluorescence intensity is observed compared to that of the free pDNA. 


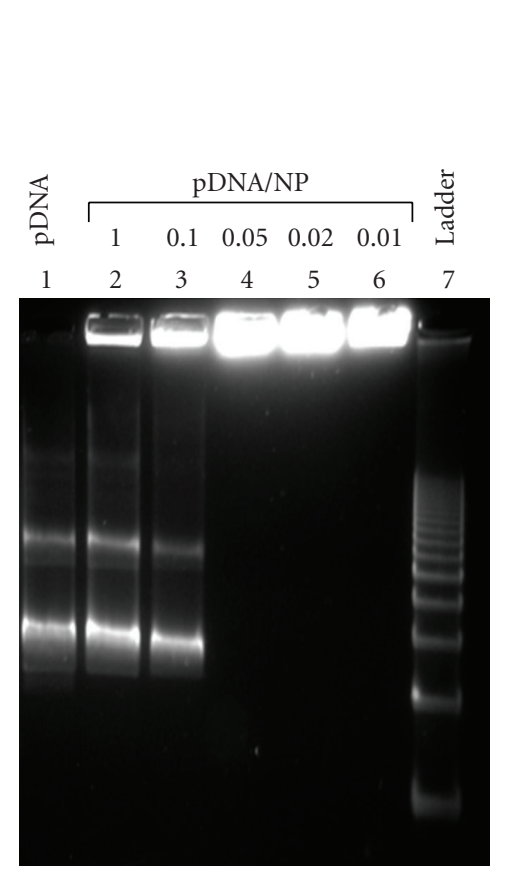

(a)

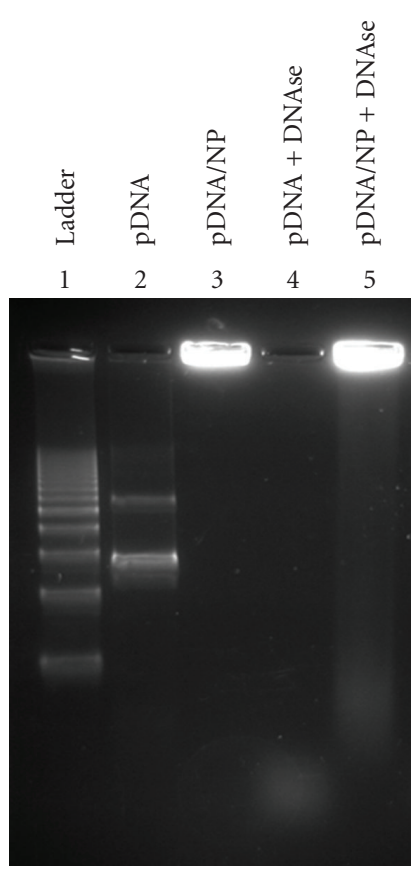

(b)

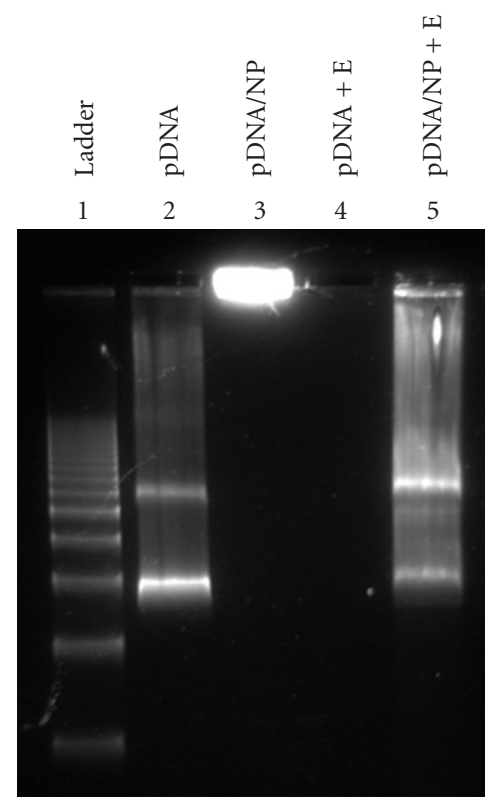

(c)

FIGURE 4: Gel analysis of complex formation and degradation. (a) Complexes formed at various ratios: lane 1: $100 \mathrm{ng}$ plasmid DNA; lane 2: 1:1 plasmid:particle complex; lane 3: 0.1; lane 4: 0.05; lane 5: 0.02; lane 6: 0.01; lane 7: $1 \mathrm{~kb}$ ladder. (b) Plasmid and pDNA/NP complexes after nuclease digestion using DNase I: lane 1: $1 \mathrm{~kb}$ ladder; lane 2: $100 \mathrm{ng}$ plasmid; lane 3: 0.02 complex; lane 4: $100 \mathrm{ng}$ plasmid with DNase I; lane 5: 0.02 complex with DNase I. (c) Plasmid and pDNA/NP complexes treated with chitosanase and lysozyme: lane 1:1 kb ladder; lane 2: 100 ng plasmid; lane 3: 0.02 plasmid:particle complex; lane 4: $100 \mathrm{ng}$ plasmid with chitosanase/lysozyme; lane 5: 0.02 complex with chitosanase/lysozyme.

Comparison of this value to a negative control containing only water indicates complete complexation of all free pDNA using these synthesis and complexing methods. The complete uptake of all of the pDNA indicates the ideal ratio for protection and delivery of the entire DNA load.

Electrophoretic mobility studies further support the Oligreen fluorescence assay data, where the mobility of free plasmids is compared to that of complexes. Plasmid bands are visible in complexes in lanes 2-3 of the gel (Figure 4(a)), indicating the presence of free pDNA in the corresponding samples of 1 and $0.1 \mathrm{pDNA} / \mathrm{NP}$ ratios, similar to the bands seen in lane 1 , that contain only free plasmid. No free plasmid bands are visible in subsequent lanes of $\mathrm{pDNA} / \mathrm{NP}$ complexes of ratios $0.05,0.02$ and 0.01 , indicating complete complexation of all free plasmid. In these samples, the only staining is visible in the gel wells, where the increased NP content retards electrophoretic mobility of the complex.

\subsection{Enzymatic Degradation of pDNA and Complexes. After} incubation with DNase I, gel electrophoresis confirms the degradation of free pDNA, with no bands visible except a faint band of high mobility at the bottom of the gel image (Figure 4(b), lane 4). The smeared pattern in lane 5 is an indication that some of the pDNA in the complex was available for digestion, as fragments of various sizes with higher mobility than those of undigested plasmid are visible. A lesser degree of digestion occurs for complexes, as the smeared band does not contain fragments as small as those in lane 4 . In the pDNA-NP sample exposed to DNase, there is still a large amount of remaining complexes that exhibit no electrophoretic mobility and are thus seen as bands in the gel well, suggesting protection from endonuclease digestion.

As a means to determine if the PLGA and chitosan nanoparticles could be digested to free plasmid DNA for further analysis, a mixture of chitosanase and lysozyme was used to digest these NPs as previously described in [22]. Gel electrophoresis of digestion of free pDNA with this enzyme mixture in this system reveals no visible pDNA bands, indicating plasmid degradation (Figure 4(c), lane 4). Comparison of lanes 3 and 5 indicates release of pDNA from complexes upon incubation with the enzyme mixture and suggests the ability of the complex to protect the DNA from enzymatic degradation. The combination of DNase and chitosanase/lysozyme digestions was used sequentially to digest free plasmid on the exterior of complexes, followed by digestion of the NP complexes to free encapsulated pDNA. The chitosanase/lysozyme was chosen to partially degrade the NP complexes so that entrapped pDNA could be released and analyzed. Because chitosanase is not an enzyme that these NPs would likely encounter in human tissue, it is obviously less relevant for modeling stability of these formulations in vivo; however this enzyme has utility in characterization studies of therapeutic formulations. In this test to treat complexes with DNase, followed by its 


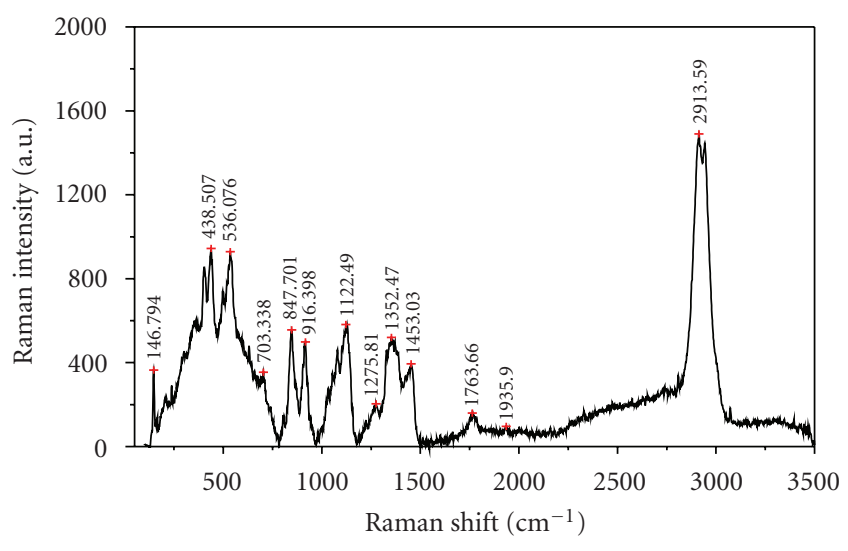

(a)

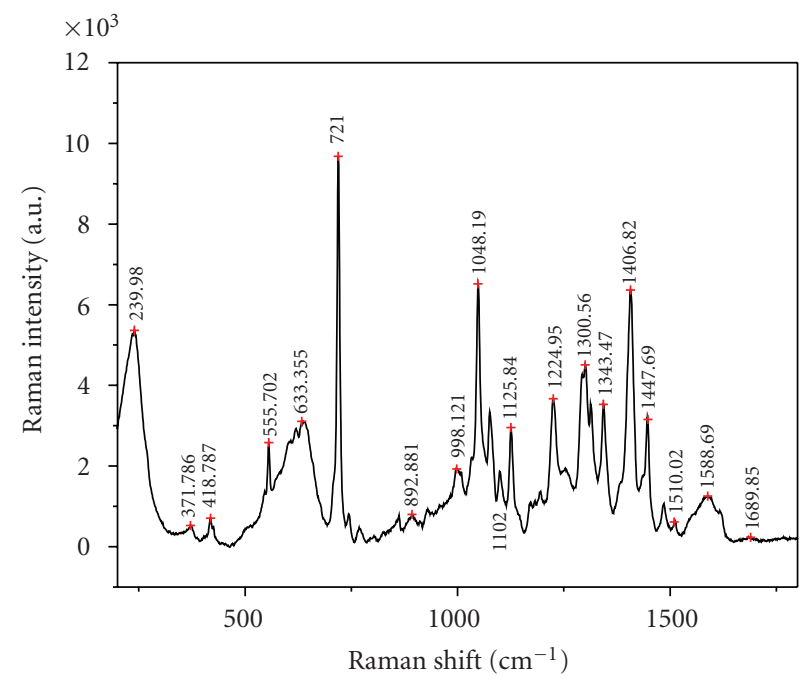

(b)

FIGURE 5: (a) Typical Raman spectrum of the pDNA/NP complexes. (b): Representative SERS spectrum of the pDNA/NP complexes conjugated PLGA NPs and decorated with silver nanoparticles (SNPs). It can be observed that the strong scattering peaks corresponding to pDNA and since the signal was mostly collected due to the SERS effect generated by the SNPs, it can be concluded that the DNA is present mostly on the surface of the PLGA NPs.

inactivation, then digestion with the chitosanase/lysozyme mixture, the result was complete digestion of the plasmid (data not shown,) suggesting either incomplete DNase inactivation or, similar to the lane 4 result, digestion of the plasmid by the chitosanase and lysozyme mixture.

4.4. Raman Spectroscopy Analysis. Raman spectroscopy is a powerful tool for the identification of various organic and biologic molecules with high specificity. One major drawback is its inability of detecting the interactions of small clusters of molecules, down to single molecule level. Organic systems, such as polymers, have strong Raman characteristic scattering features, which can overlap onto the peaks corresponding to biological molecules, like DNA, making the spectral identification and distinction between

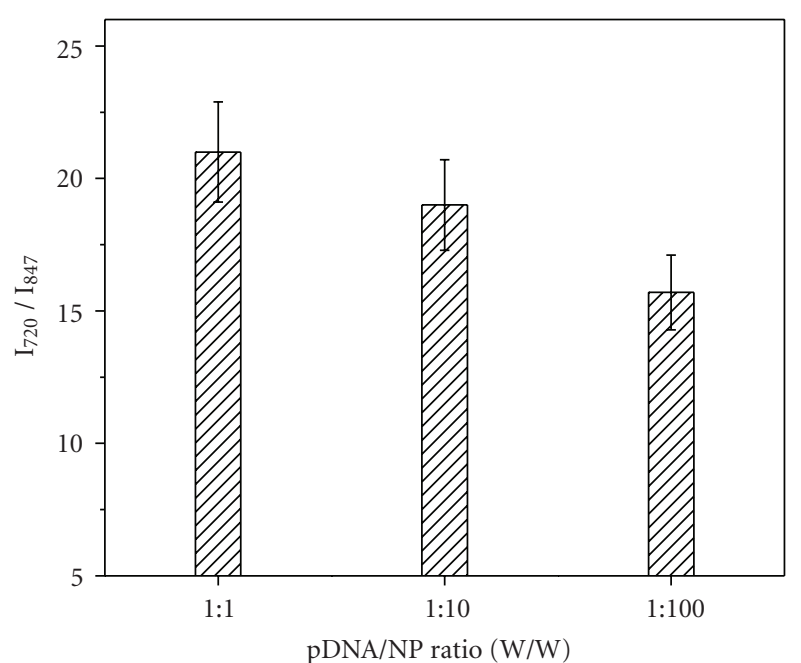

FIGURE 6: The variation of the ratio between the $720 \mathrm{~cm}^{-1}$ (DNAAdenine) and $847 \mathrm{~cm}^{-1}$ PLGA ( $\mathrm{rCH} 2$ ) peaks intensity values with the relative concentration of DNA to polymer NPs based on the SERS analysis of the complexes.

such systems difficult. A characteristic Raman spectrum of the pDNA-NP complexes is shown in Figure 5(a) and is typical to the polymeric structures (with the strongest peak features corresponding to the $\mathrm{CH}$ stretching modes $\left.2900-3000 \mathrm{~cm}^{-1}\right)$. The $847 \mathrm{~cm}^{-1}$ peak ( $\left.\mathrm{rCH} 2\right)$ was considered as a reference band for the concentration analysis of the polymer NP relative to pDNA. Very weak peaks overimposed onto the polymer spectrum can be attributed to pDNA. Given the depth of penetration of the $633 \mathrm{~nm}$ laser beam into the polymeric samples, the presence of the pDNA strands onto the surface of the polymeric nanostructures is difficult to prove and quantify, since their corresponding peaks are significantly weaker as compared to those of the polymer NPs. As a result, a novel SERS-based approach was used to only acquire Raman signal from the top surface of the polymer surfaces. For this, silver nanoparticles (SNPs) were deposited onto the surfaces of the pDNA-PLGA complexes, which enabled a strong enhancement of the pDNA signal, as shown in Figure 5(b). The presence of the SNPs on the surfaces of the polymer NPs was found to be responsible for a strong SERS effect, which preferentially allowed the collection of Raman scattering signal from the surface of the pDNA-coated polymer nanostructures (the peaks corresponding to the polymer nanomaterials were highly reduced or disappeared, including the strong $\mathrm{C}-\mathrm{H}$ stretching modes; not shown here). The characteristic $721 \mathrm{~cm}^{-1}$ peaks assigned to Adenine are easily visible, similar to that of both Raman and silver-based SERS studies on DNA [29, 30]. This approach allowed the understanding, with clear identification and probing, of the surface morphology and chemistry of the polymer NPs by indicating that while much of the pDNA is entrapped within the complexes, some of the DNA molecules are present on the outer surface. Further morphological studies will be necessary to determine the exact conformation of the exterior pDNA on outer complex 
surfaces, yet it is presumed that it is not completely sterically shrouded by polymer, as some enzymatic degradation of the plasmid does still occur.

The strong SERS effect generated by the SNPs deposited onto the surface of the pDNA-NP complexes was also used to obtain relative pDNA concentration information relative to the polymer NPs as their relative concentration was varied in the following pDNA/NP ratios: $1,0.1$ and 0.01 . The results are presented in Figure 6 . It can be clearly observed that as the ratio $\mathrm{pDNA} /$ polymer decreases, the ratios between the $721 \mathrm{~cm}^{-1}$ (pDNA) and $847 \mathrm{~cm}^{-1}$ (PLGA) peaks follow a similar decreasing trend. Therefore, it can be concluded that the pDNA coverage of the NP complexes surface varied according to their relative concentrations.

\section{Discussion}

Complex formation between chitosan-PLGA NPs and pDNA was confirmed using both fluorescence and gel electrophoresis techniques. Significant complex formation was seen beginning in pDNA/NP ratios of 0.1 and lower. Using the fluorescent intercalating dye OliGreen, free pDNA within the complexes could be labeled and quantified relative to samples containing only free pDNA. The complexation of all pDNA was confirmed by comparison of the relative fluorescence of samples containing pDNA-NP complexes (0.02 ratio) to a pure water sample and indicates that no free pDNA is present. The lack of electrophoretic mobility of the pDNA in the complexed form further confirms complete encapsulation, as no free plasmid bands were visible in complexes with $\mathrm{pDNA} / \mathrm{NP}$ ratios lesser than 0.1 . These data suggest good correlation between the fluorescence staining method and the gel mobility method, both of which are relatively easy to carry out.

The protection from enzymatic digestion that NP complexes afford to pDNA was confirmed by gel electrophoresis after treatment with DNase and chitosanase/lysozyme mixtures. A standard pDNA/NP complex ratio of 0.02 was subjected to enzyme digestion and gel analysis. Partial complex dissociation was seen with treatment of chitosanase and lysozyme, where gel electrophoresis confirms that no free pDNA is present after initial complex formation. Chitosanase/lysozyme digestion degrades the nanoparticle portion of the complexes, freeing the pDNA. Gel digest studies also confirm that free pDNA is unable to sustain exposure to the concentrated chitosanase/lysozyme solution and is completely degraded. The presence of intact pDNA in the 0.02 complex sample suggests the ability of the NPs to provide protection from enzymatic degradation, an aspect important for efficient gene delivery in vivo.

The addition of DNase I to free pDNA was used as a control to measure the extent of free plasmid degradation. Complexes treated with the nuclease exhibit limited electrophoretic mobility, indicating that within the complex, some of the pDNA must be exposed to the introduced enzyme. Because the complex offers protection from enzymatic degradation to the pDNA within it, this data suggests that the degraded DNA is located on the outside of the complex. These results are in excellent correlation with the SERS analysis afforded by SNPs deposited on surface of pDNA/NP complexes. Standard Raman analysis of the complexes did not enable elucidation of DNA peaks within the NP complex, yet silver-based SERS showed greatly enhanced DNA peaks and clearly indicated the presence of pDNA strands on the surface of the complexes. Such a dual approach for the surface analysis of polymeric nanoparticles complexed with various biological systems could represent a technique not limited for the study of their chemical and morphological properties but useful also for following them in vitro and in vivo as they interact with various cells and tissues. Such approaches could also prove useful in studying the delivery of the biological agents into cells via nanoparticle platforms and the release of the active therapeutics from polymeric nanosurfaces.

\section{Conclusions}

PLGA-chitosan NPs $150 \mathrm{~nm}$ in size, with a zeta potential of $+34 \mathrm{mV}$, were synthesized by the proposed, optimized method. Complexing of NPs with pDNA was successful, with pDNA-NP complexes less than $200 \mathrm{~nm}$ in size for $0.025,0.05$, $0.01,0.02,0.1$, and $1.0 \mathrm{pDNA} / \mathrm{NP} \mathrm{w} / \mathrm{w}$ ratios. All complexes were positively charged for the ratios studied $(>20 \mathrm{mV})$. Complexes with pDNA/NP ratios of 0.02 and greater showed no electrophoretic mobility, indicating the successful uptake of all pDNA into the complex. Electrophoresis studies of the complexes after incubation with chitosanase/lysozyme indicate their ability to offer protection of the pDNA from enzymatic degradation. This characteristic is of importance for clinical adoption of these formulations, where protection of nucleic acids from enzymatic degradation in both the extracellular and intracellular spaces is critical for in vivo efficacy. The electrophoretic mobility of the complexes after treatment with DNAse I suggests that some of the pDNA is located on the outside of the complex, and SERS data supports these findings. The decoration of the pDNA-NP complexes with SNPs allowed the use of Raman spectroscopy for the analysis of the surface-only micro environments of the samples and indicates the presence of exposed pDNA strands at the surface of the polymer NP structures. This novel nondestructive approach can be developed in highly sensitive platforms for understanding and studying the interactions between biologic and organic systems. Clarification of the location of DNA within polymeric nanoparticulate delivery systems will aid in optimizing formulations that can achieve targeted delivery with minimal degradation. These aspects will be critical for good manufacturing practices in pharmaceutical development, validation, and clinical administration of these therapeutics. Further studies are needed to evaluate the in vitro and in vivo delivery and expression of plasmids in this form of the PLGA-chitosan NP complex and whether these results correlate with the variety of physicochemical characterizations made in this study.

\section{Acknowledgments}

The authors would like to thank Anna Charron Dugas and Meocha Whaley at Louisiana State University for technical 
expertise in particle synthesis. Funding was provided by the National Science Foundation (CBET-0748195) and the State of Louisiana Board of Regents Support Fund. This material is based upon work supported by an Honors College TAF grant to $\mathrm{H}$. Bordelon. This paper was approved for publication by the Director of the Louisiana Agricultural Experiment Station as no. 2010-232-5138. C. M. Sabliov and W. T. Monroe contributed equally to this work.

\section{References}

[1] S. S. Davis, "Biomedical applications of nanotechnologyimplications for drug targeting and gene therapy," Trends in Biotechnology, vol. 15, no. 6, pp. 217-224, 1997.

[2] T. Niidome and L. Huang, "Gene therapy progress and prospects: nonviral vectors," Gene Therapy, vol. 9, no. 24, pp. 1647-1652, 2002.

[3] E. J. Simon, "Human gene therapy: genes without frontiers?" American Biology Teacher, vol. 64, no. 4, pp. 264-270, 2002.

[4] J. Zhu, A. Tang, L. P. Law et al., "Amphiphilic core-shell nanoparticles with poly(ethylenimine) shells as potential gene delivery carriers," Bioconjugate Chemistry, vol. 16, no. 1, pp. 139-146, 2005.

[5] J. Panyam and V. Labhasetwar, "Biodegradable nanoparticles for drug and gene delivery to cells and tissue," Advanced Drug Delivery Reviews, vol. 55, no. 3, pp. 329-347, 2003.

[6] S. M. Moghimi, A. C. Hunter, and J. C. Murray, "Nanomedicine: current status and future prospects," FASEB Journal, vol. 19, no. 3, pp. 311-330, 2005.

[7] A. Bragonzi, A. Boletta, A. Biffi et al., "Comparison between cationic polymers and lipids in mediating systemic gene delivery to the lungs," Gene Therapy, vol. 6, no. 12, pp. 19952004, 1999.

[8] W. R. Gombotz and D. K. Pettit, "Biodegradable polymers for protein and peptide drug delivery," Bioconjugate Chemistry, vol. 6, no. 4, pp. 332-351, 1995.

[9] I. Bala, S. Hariharan, and M. N. V. R. Kumar, "PLGA nanoparticles in drug delivery: the state of the art," Critical Reviews in Therapeutic Drug Carrier Systems, vol. 21, no. 5, pp. 387-422, 2004.

[10] M. Koping-Hoggard, I. Tubulekas, H. Guan et al., "Chitosan as a nonviral gene delivery system. Structure-property relationships and characteristics compared with polyethylenimine in vitro and after lung administration in vivo," Gene Therapy, vol. 8, no. 14, pp. 1108-1121, 2001.

[11] M. X. Tang and F. C. Szoka, "The influence of polymer structure on the interactions of cationic polymers with DNA and morphology of the resulting complexes," Gene Therapy, vol. 4, no. 8, pp. 823-832, 1997.

[12] D. Goula, C. Benoist, S. Mantero, G. Merlo, G. Levi, and B. A. Demeneix, "Polyethylenimine-based intravenous delivery of transgenes to mouse lung," Gene Therapy, vol. 5, no. 9, pp. 1291-1295, 1998.

[13] M. N. V. R. Kumar, U. Bakowsky, and C. M. Lehr, "Preparation and characterization of cationic PLGA nanospheres as DNA carriers," Biomaterials, vol. 25, no. 10, pp. 1771-1777, 2004.

[14] M. N. V. R. Kumar, S. S. Mohapatra, X. Kong, P. K. Jena, U. Bakowsky, and C.-M. Lehr, "Cationic poly(lactide-coglycolide) nanoparticles as efficient in vivo gene transfection agents," Journal of Nanoscience and Nanotechnology, vol. 4, no. 8, article no. 005, pp. 990-994, 2004.

[15] A. Bozkir and O. M. Saka, "Chitosan nanoparticles for plasmid DNA delivery: effect of chitosan molecular structure on formulation and release characteristics," Drug Delivery, vol. 11, no. 2, pp. 107-112, 2004.

[16] Y. Zhang, J. Chen, Y. Zhang et al., "A novel PEGylation of chitosan nanoparticles for gene delivery," Biotechnology and Applied Biochemistry, vol. 46, no. 3-4, pp. 197-204, 2007.

[17] T. Dastan and K. Turan, "In vitro characterization and delivery of chitosan-DNA microparticles into mammalian cells," Journal of Pharmacy and Pharmaceutical Sciences, vol. 7, no. 2, pp. 205-214, 2004.

[18] S. P. Kasturi, K. Sachaphibulkij, and K. Roy, "Covalent conjugation of polyethyleneimine on biodegradable microparticles for delivery of plasmid DNA vaccines," Biomaterials, vol. 26, no. 32, pp. 6375-6385, 2005.

[19] K. Tahara, T. Sakai, H. Yamamoto, H. Takeuchi, and Y. Kawashima, "Establishing chitosan coated PLGA nanosphere platform loaded with wide variety of nucleic acid by complexation with cationic compound for gene delivery," International Journal of Pharmaceutics, vol. 354, no. 1-2, pp. 210-216, 2008.

[20] X.-P. Guan, D.-P. Quan, K.-R. Liao, W. Tao, X. Peng, and K.-C. Mai, "Preparation and characterization of cationic chitosan-modified poly(D,L-lactide-co-glycolide) copolymer nanospheres as DNA carriers," Journal of Biomaterials Applications, vol. 22, no. 4, pp. 353-371, 2008.

[21] J. Chen, B. Tian, X. Yin et al., "Preparation, characterization and transfection efficiency of cationic PEGylated PLA nanoparticles as gene delivery systems," Journal of Biotechnology, vol. 130, no. 2, pp. 107-113, 2007.

[22] H.-Q. Mao, K. Roy, V. L. Troung-Le et al., "Chitosan-DNA nanoparticles as gene carriers: synthesis, characterization and transfection efficiency," Journal of Controlled Release, vol. 70, no. 3, pp. 399-421, 2001.

[23] K. Khatri, A. K. Goyal, P. N. Gupta, N. Mishra, and S. P. Vyas, "Plasmid DNA loaded chitosan nanoparticles for nasal mucosal immunization against hepatitis B," International Journal of Pharmaceutics, vol. 354, no. 1-2, pp. 235-241, 2008.

[24] N. Nafee, S. Taetz, M. Schneider, U. F. Schaefer, and C.-M. Lehr, "Chitosan-coated PLGA nanoparticles for DNA/RNA delivery: effect of the formulation parameters on complexation and transfection of antisense oligonucleotides," Nanomedicine, vol. 3, no. 3, pp. 173-183, 2007.

[25] S. Taetz, N. Nafee, J. Beisner et al., "The influence of chitosan content in cationic chitosan/PLGA nanoparticles on the delivery efficiency of antisense 2'-O-methyl-RNA directed against telomerase in lung cancer cells," European Journal of Pharmaceutics and Biopharmaceutics, vol. 72, no. 2, pp. 358369, 2008.

[26] H. Katas, S. Chen, A. A. Osamuyimen, E. Cevher, and H. O. Alpar, "Effect of preparative variables on small interfering RNA loaded Poly(D,L-lactide-co-glycolide)-chitosan submicron particles prepared by emulsification diffusion method," Journal of Microencapsulation, vol. 25, no. 8, pp. 541-548, 2008.

[27] S. Mao, W. Sun, and T. Kissel, "Chitosan-based formulations for delivery of DNA and siRNA," Advanced Drug Delivery Reviews, vol. 62, no. 1, pp. 12-27, 2009.

[28] R. B. Gupta, "Nanoparticle technology for drug delivery," in Drugs and the Pharmaceutical Sciences: Nanoparticle Technology for Drug Delivery, vol. 159, p. 403, Informa Health Care, 2006.

[29] T. O’Connor, S. Mansy, M. Bina, D. R. McMillin, M. A. Bruck, and R. S. Tobias, "The pH-dependent structure of calf thymus DNA studied by Raman spectroscopy," Biophysical Chemistry, vol. 15 , no. 1, pp. 53-64, 1982. 
[30] F. Feng, G. Zhi, H. S. Jia, L. Cheng, Y. T. Tian, and X. J. Li, "SERS detection of low-concentration adenine by a patterned silver structure immersion plated on a silicon nanoporous pillar array," Nanotechnology, vol. 20, no. 29, Article ID 295501, 2009. 

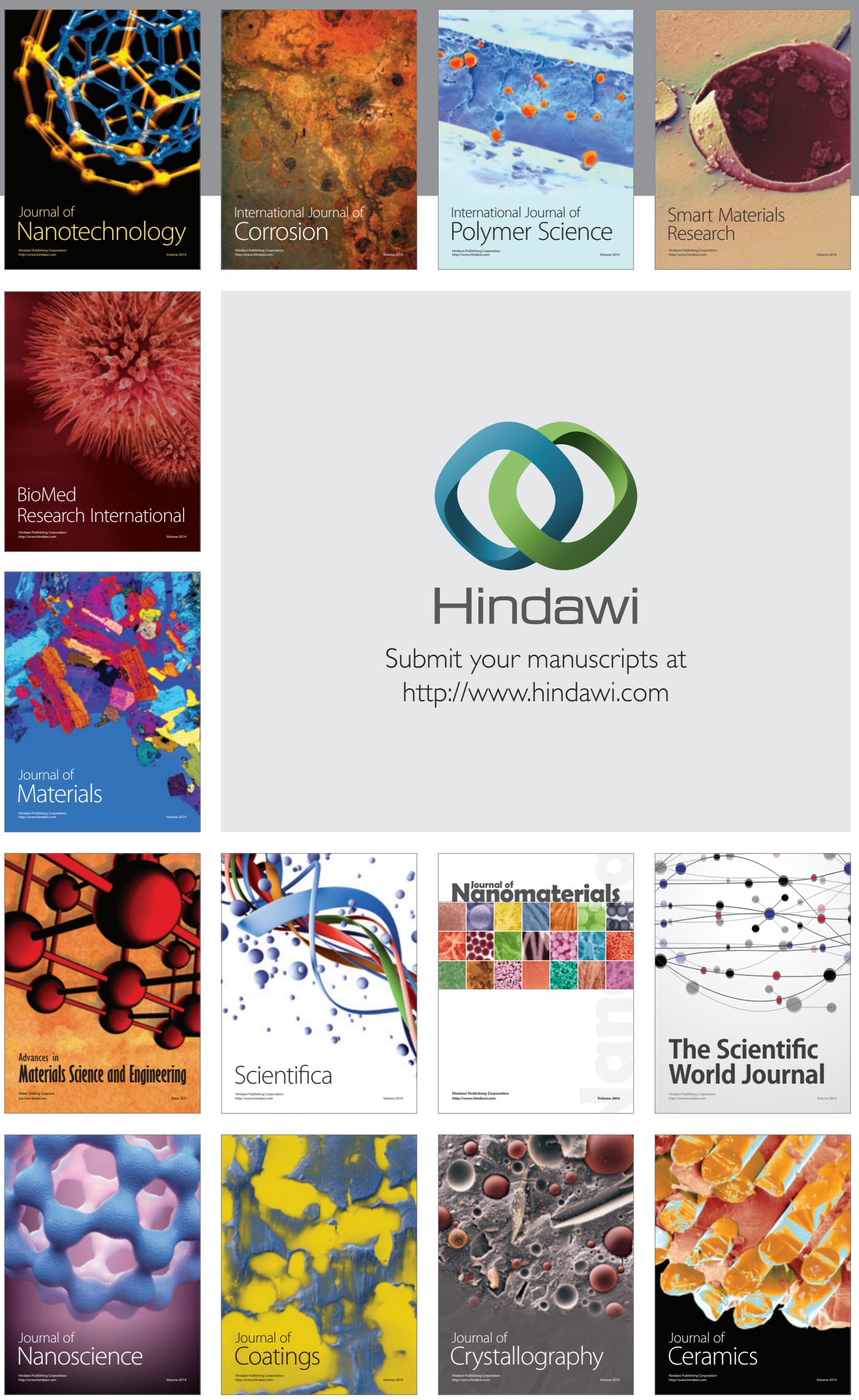

The Scientific World Journal

Submit your manuscripts at

http://www.hindawi.com

\section{World Journal}

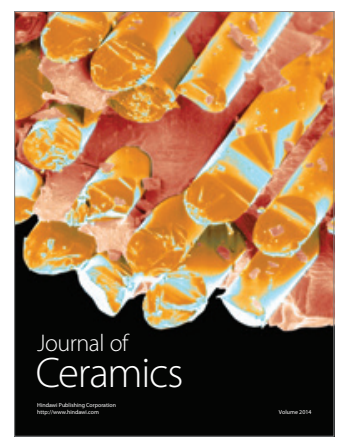

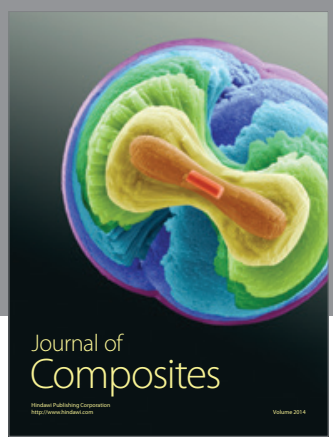
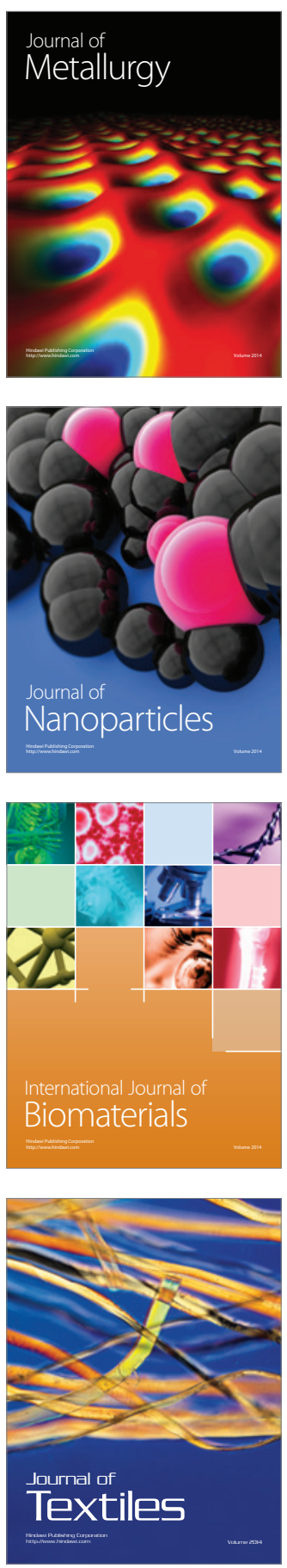